\title{
Statins and Diabetes: The Plot Thickens
}

\author{
Giacomo Gastaldi, MD and Jacques Philippe, MD
}

Division of Endocrinology, Diabetology, Hypertension and Nutrition, Geneva University Hospital, Geneva, Switzerland

J Gen Intern Med 30(11):1572-3

DOI: $10.1007 / \mathrm{s} 11606-015-3399-y$

(c) Society of General Internal Medicine 2015

$\mathrm{S}$ tatins have now been used in clinical practice for more than 20 years, and are the cornerstone of primary and secondary prevention of cardiovascular disease. The increased risk of new-onset type 2 diabetes in patients treated with statins has been well established by large meta-analyses of randomized controlled trials and observational studies. $^{1,2}$

In a meta-analysis of 13 statin intervention trials involving 91,140 participants, statin therapy was associated with a $9 \%$ increased risk of incident diabetes; treatment of 255 patients with statins for 4 years resulted in one extra case of diabetes, while 5.4 cases of death or myocardial infarct were prevented. ${ }^{1}$ Similar results were obtained from another meta-analysis comparing intensive-dose with moderate-dose statin therapy among 32,752 participants without diabetes; 2.0 additional cases of diabetes in the statin-intensive group were observed per 1000 patient-years, but with 6.5 fewer instances of cardiovascular events. ${ }^{3}$

Recent data from the higher-risk population cohort in the Metabolic Syndrome in Men (METSIM) study, however, which included 8749 non-diabetic participants aged 45 to 73 years who were followed for 5.9 years, indicated a $56 \%$ increased risk (11.2 versus $5.8 \%$ ) in statin-treated patients, which was dose-dependent for simvastatin and atorvastatin, suggesting that statin treatment may be associated with a much higher risk of diabetes in a population with a high risk of diabetes at baseline. ${ }^{4}$

Even in patients with type 2 diabetes, statin treatment is associated with worsening glycemic control. In pooled analyses of nine trials involving 9696 patients over a follow-up period of 3.6 years, mean HbA1c levels increased by $0.2 \%$ in patients randomized to statin versus control treatment. ${ }^{5}$

This dysglycemic effect of statins is apparently due to a reduction in both insulin sensitivity and secretion. ${ }^{4}$ In addition, variants in the gene encoding HMG-CoA reductase, the target of statins, are associated with higher

Published online May 19, 2015 body weight, waist circumference, plasma glucose, and insulin concentrations, suggesting that the increased risk of type 2 diabetes due to statins may be partially explained by HMG-CoA reductase inhibition. ${ }^{6}$ Furthermore, studies have suggested that statin treatment is related to impaired exercise training and decreased diet compliance. ${ }^{7}$

A new dimension in this saga is suggested in this issue of JGIM by Mansi et al., who report that statin use may be associated with diabetic complications in a retrospective cohort study of adult patients enrolled in the US military's Tricare program in San Antonio, TX. ${ }^{8}$ The authors selected patients without cardiovascular problems or significant comorbid conditions and, using propensity scores to match the two groups, selected 3351 statin users and 3351 non-users. After follow-up of 6.5 years, the authors observed that statin users had an odds ratio (OR) of 1.87 for diabetes (30.9\% versus $19.4 \%), 2.5$ for diabetic complications (5.0\% versus $2.1 \%$ ), and 1.14 for overweight/obesity (46.7\% versus $43.4 \%$ ) compared to non-users. Furthermore, the adjusted ORs for high-intensity statin users and 4-year users were higher than those for moderate/low-intensity users and 2-year users, respectively. More specifically, uncontrolled diabetes and renal, ophthalmic, and neurological complications, as well as peripheral circulation problems, were significantly increased in statin users. The increased risk of diabetes observed in this study is considerably higher than that in the previously mentioned meta-analyses ${ }^{1}$ and is similar to that reported in the METSIM study. ${ }^{4}$ In addition, the risk is dose- and exposure-dependent, in agreement with previous reports, ${ }^{2,3}$ which strengthens the case for a causal role of statins. Similar to the results in previous studies, the authors also report an association with overweight and obesity. ${ }^{6,7}$ Although this study adds significant information on the relationship between statins and diabetes, it has several important limitations. These include the retrospective observational design, the baseline classification of patients based on disease codes, and the lack of detailed cardiovascular risk factors and laboratory variables. Importantly, diabetic complications are not defined and are very low in number.

The results of the study should be interpreted in light of current knowledge. In a recent meta-analysis, 
statin treatment in patients with type 2 diabetes was shown to lead to a modest increase of $0.12 \%$ in HbA1c levels compared to controls over a 3.6-year follow-up period, ${ }^{5}$ although details were missing on the statin drugs and dosages as well as on whether antidiabetic treatment was more aggressive in statin users, two parameters that may have contributed to underestimation of the increase in HbAlc. However, this small change is unlikely to have a significant effect on the incidence or severity of diabetic complications (particularly cardiovascular events) in newly diagnosed diabetic patients. Furthermore, we have for years employed treatments such as thiazide diuretics and beta-blockers-drugs which also cause dysglycemia, but with fewer benefits in terms of cardiovascular prevention compared to statins.

The study by Mansi et al. ${ }^{8}$ calls for more consideration of the consequences of statin treatment, particularly in primary prevention for patients at risk of developing diabetes (overweight/obese older individuals), and potentially on the development of microangiopathic complications, although these risks are far from established on the basis of this study and should be the subject of more rigorous prospective trials. Physicians should not alter their current practices based on this study, as the benefit of statin treatment on cardiovascular events has been rigorously demonstrated in multiple prospective intervention studies in diabetic patients over the last 20 years. Rather, this paper should stimulate more research efforts to better assess the over- all benefit of statin treatment in diabetes and in patients at high risk of developing diabetes.

Conflict of Interest: The authors declare that they do not have a conflict of interest.

Corresponding Author: Jacques Philippe, MD; Division of Endocrinology, Diabetology, Hypertension and NutritionGeneva University Hospital, Geneva, Switzerland (e-mail: jacques.philippe@hcuge.ch).

\section{REFERENCES}

1. Sattar N, Preiss D, Murray HM, et al. Statins and risk of incident diabetes: a collaborative meta-analysis of randomized statin trials. Lancet. 2010;27:735-742.

2. Dormuth CR, Filion KB, Parerson JM, et al. Higher potency statins and the risk of the diabetes: multicentre, observational study of administrative databases. BMJ. 2014;348:g3244.

3. Preiss D, Kondapally Seshasai SR, Welsh P, et al. Risk of incident diabetes with intensive-dose compared with moderate-dose statin therapy: a metaanalysis. JAMA. 2011;305(24):2556-2564.

4. Cederberg $\mathbf{H}$, Stancakova A, Yaluri $\mathbf{N}$, et al. Increased risk of diabetes with statin treatment is associated with impaired insulin sensitivity and insulin secretion: a 6 year follow-up study of the METSIM cohort. Diabetologia. 2015;s00125-015:3528-5.

5. Erqou S, Lee CC, Adler AI. Statins and glycaemic control in individuals with diabetes: a systematic review and meta-analysis. Diabetologia. 2014;57:2444-2452

6. Swerdlow D, Preiss D, Kuchenbaecker KB, et al. HMG-coenzyme a reductase inhibition, type 2 diabetes, and bodyweight: evidence from genetic analysis and randomized trials. Lancet. 2014;S0140-6736(14):61183-1.

7. Radford NB, DeFina LF, Barlow CE, et al. Effect of fitness on incident diabetes from statin use in primary prevention. Atherosclerosis. 2015;239:43-49.

8. Mansi I, Frei CR, Wang CP, Mortensen EM. Statins and new-onset diabetes mellitus and diabetic complications: a retrospective cohort study of US healthy adults. J Gen Int Med doi: 10.1007/s11606-015-3335-1 